\title{
Application of Complementary Error Function to the Transition Zone of Myopic Ablation Shapes in Refractive Surgery
}

\author{
Guang-Ming Dai \\ Research and Development, Johnson and Johnson Vision, Milpitas, CA, USA \\ Email:gdai5@its.jnj.com
}

How to cite this paper: Dai, G.-M. (2017) Application of Complementary Error Function to the Transition Zone of Myopic Ablation Shapes in Refractive Surgery. Journal of Applied Mathematics and Physics, 5, 1521-1528.

https://doi.org/10.4236/jamp.2017.58125

Received: March 16, 2017

Accepted: July 31, 2017

Published: August 4, 2017

Copyright $\odot 2017$ by author and Scientific Research Publishing Inc. This work is licensed under the Creative Commons Attribution International License (CC BY 4.0).

http://creativecommons.org/licenses/by/4.0/

\begin{abstract}
In refractive surgery, the cubic spline fit for the transition zone breaks down for myopia and myopic meridians in mixed astigmatism as in many cases the cubic spline function runs into negative values. In this paper, the complementary error function is proposed instead of the cubic spline function as the transition zone function, due to the availability of analytical expression of its derivatives and the nonnegativity fact. It is shown that with the use of the complementary error function, transition zones for all refractive types work correctly.
\end{abstract}

\section{Keywords}

Error Function, Complementary Error Function, Vision Correction, Refractive Surgery, Treatment Algorithm

\section{Introduction}

In refractive surgery [1], where human tissue is ablated to correct for vision, a central area called the optical zone (OZ) is designed such that the optics within the zone is optimal for vision. However, the surrounding area, which is called the transition zone (TZ), needs serious consideration; or there might be associated vision problems such as glares, star bursts, halos, to name a few, due to abrupt curvature changes. Traditionally, cubic spline functions are used to perform the task for transitioning the ablation area to the unablated corneal surface.

The spline functions have been widely used for applications in various fields, such as for smoothing [2] [3], data analysis [4], curve fitting [5] [6], image mosaic [7], and in vision [8] [9]. When two surface zones need to be connected, discontinuity between the two zones is a major concern [10]. For our application, 
where a nonzero area is to be transitioned to zero, geometric continuity and parametric continuity are considered. For geometric continuity, a nonzero value needs to be transitioned to zero; for parametric continuity, surface slope (first derivative) must be smoothly connected.

The cubic spline functions work correctly for refractive surgical types such as hyperopia and hyperopic astigmatism. However, for myopia, myopic astigmatism and mixed astigmatism, the transition zone runs into negative values when the edge of the optical zone is too steep (slope smaller than -2). Since no negative tissue can be ablated, negative values are usually ignored, causing incorrect ablation shapes.

In this paper, complementary error function is used as the transition zone function for cases when the slope of the optical zone edge is lower than -2 . The geometric continuity and the parametric continuity for the complementary error function are discussed. Simulation of the real-life ablation shapes with the use of both cubic spline function and complementary error function is given to show the effectiveness for the use of the complementary error function.

\section{Review of Cubic Spline Fit Mathematics}

For simplicity, assume that the transition zone starts from $u=0$ to $u=1$ along any radial meridian for a two-dimensional surface. Further, assume that the transition zone function has values between 0 and 1, shown in Figure 1, as it is easy to be multiplied to scale the transition zone values.

The general form for a cubic function can be given by

$$
S(u)=a u^{3}+b u^{2}+c \cdot u+d,
$$

where $a, b, c$, and $d$ are the unknowns. We further assume that the slope at the outer edge of the optical zone is $s$. With these four unknowns, it is necessary to use four equations to solve them. Generally speaking, these are the four conditions that form the four equations:
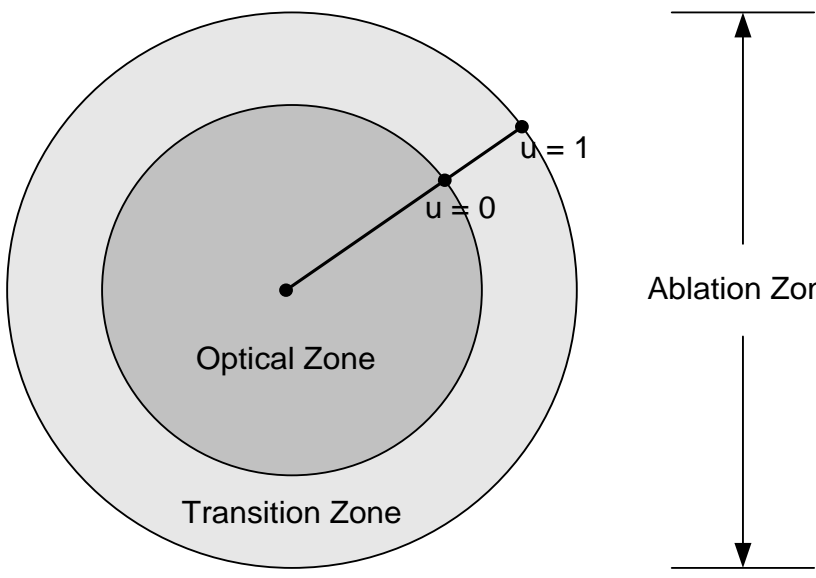

Ablation Zone

Figure 1. Schematic of the optical zone (OZ), transition zone (TZ) and ablation zone (AZ). Each radial line is considered as a cross section for a given meridian. 
- When $u=0, S(u)=1$ for geometric continuity at the inner edge;

- When $u=1, S(u)=0$ for geometric continuity at the outer edge;

- When $u=0, S^{\prime}(u)=\mathrm{s}$ for parametric (slope) continuity at the inner edge;

- When $u=1, S^{\prime}(u)=0$ for parametric (slope) continuity at the outer edge.

Using the four equations obtained from these conditions yields the solution as

$$
S(u)=(s+2) u^{3}-(2 s+3) u^{2}+s \cdot u+1 .
$$

This solution works correctly when the slope at the outer edge of the optical zone $(s)$ is greater than -2 . However, when $s<-2$, Equation (2) has the potential to result in a negative value, since the first derivative of $S(u)$ in Equation (2) is quadratic and it has two roots. If the first one occurs at $u=1$, the other one may occur at $u<1$. When this happens, the transition zone contains negative values. Since no negative ablations can be allowed, they must be zeroed out, resulting in the implemented transition zone much smaller, as illustrated in Figure 2.

To prevent negative values to occur, we can force $S(u)$ to be always positive, thus

$$
(s+2) u^{3}-(2 s+3) u^{2}+s \cdot u+1 \geq 0,
$$

yielding

$$
s \geq-\frac{2 u^{3}-3 u^{2}+1}{u^{3}-2 u^{2}+u}
$$

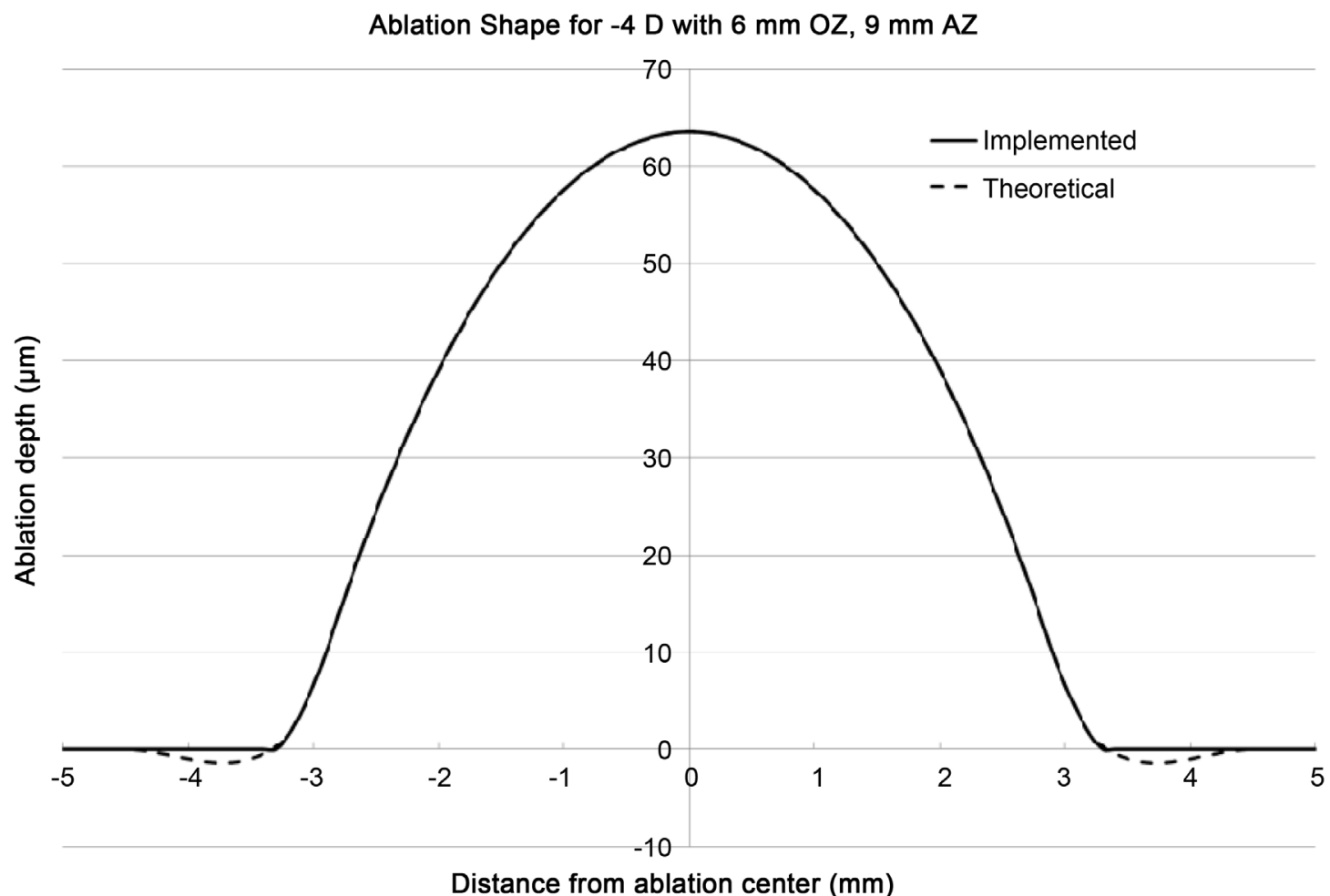

Figure 2. Cross sections of ablation profile for a $-4 \mathrm{D}$ with a $6 \mathrm{~mm} \mathrm{OZ}$ and $9 \mathrm{~mm} \mathrm{AZ}$. The theoretical value of the transition zone at $u=1$ is 0 and the slope at $u=1$ is also 0 . But since the transition zone has negative values, they were zeroed out, resulting in an effective ablation zone of $6.5 \mathrm{~mm}$. 
It is easy to show that the asymptotic value of $s$ is -2 :

$$
\lim _{u \rightarrow \infty} s=\lim _{u \rightarrow \infty}\left[-\frac{2 u^{3}-3 u^{2}+1}{u^{3}-2 u^{2}+u}\right]=-2 .
$$

Based on the above analysis, it is necessary to use a different transition function for situations when the slope is smaller than -2 , to prevent running into negative transition zone values.

\section{Complementary Error Function as a Transition Zone Function}

From the fact that the ideal transition zone function spans from 1 to 0 without resulting in negative values, it is easy to think about the complementary error function, which runs from 1 to 0 asymptotically. It will never run into negative values. The complementary error function is given by

$$
\operatorname{erfc}(u)=1-\operatorname{erf}(u)=1-\frac{2}{\sqrt{\pi}} \int_{0}^{u} \mathrm{e}^{-t^{2}} \mathrm{~d} t=\frac{2}{\sqrt{\pi}} \int_{u}^{\infty} \mathrm{e}^{-t^{2}} \mathrm{~d} t
$$

It is an important property that the complementary error function has an analytical form of its derivative, which is critical for laser vision correction application. Its derivative is given by

$$
\frac{\partial \operatorname{erfc}(u)}{\partial u}=-\frac{2}{\sqrt{\pi}} \mathrm{e}^{-u^{2}}
$$

Figure 3 shows both the complementary error function and its derivative. If we normalize the derivative by the complementary error function, we get the normalized derivative of the complementary error function as

$$
D(u)=\frac{\frac{\partial \operatorname{erfc}(u)}{\partial u}}{\operatorname{erfc}(u)}=-\frac{\mathrm{e}^{-u^{2}}}{\int_{u}^{\infty} \mathrm{e}^{-t^{2}} \mathrm{~d} t}
$$

An approximation of Equation (8) may be obtained [11] as

$$
D(u)=-\frac{2 u}{1-\frac{1}{2 u^{2}}+\frac{1 \times 3}{\left(2 u^{2}\right)^{2}}-\frac{1 \times 3 \times 5}{\left(2 u^{2}\right)^{3}}+\frac{1 \times 3 \times 5 \times 7}{\left(2 u^{2}\right)^{4}}-\frac{1 \times 3 \times 5 \times 7 \times 9}{\left(2 u^{2}\right)^{5}}+\cdots}
$$

Note the normalized derivative of the complementary error function is very close to a straight line, as shown in Figure 4 . If we take only one term in the denominator in Equation (9), we obtain a very simple approximation: $D(u)=-2 u$. Equation (8) can be used to calculate where to start the normalized complementary error function, defined as

$$
S(u)=\frac{\operatorname{erfc}\left(u_{0}+u\right)}{\operatorname{erfc}\left(u_{0}\right)} .
$$

Here, $u_{0}$ can be calculated by using $D(u)=s$. This property is very useful for the implementation of $S(u)$ as the transition zone function. Therefore, at $u_{0}$, the derivative of $S(u)$ equals to $s$ when $u=0$. When $u=1, S(u)$ gets close to zero. In- 
deed, $S(u)$ can be used as a transition zone function.

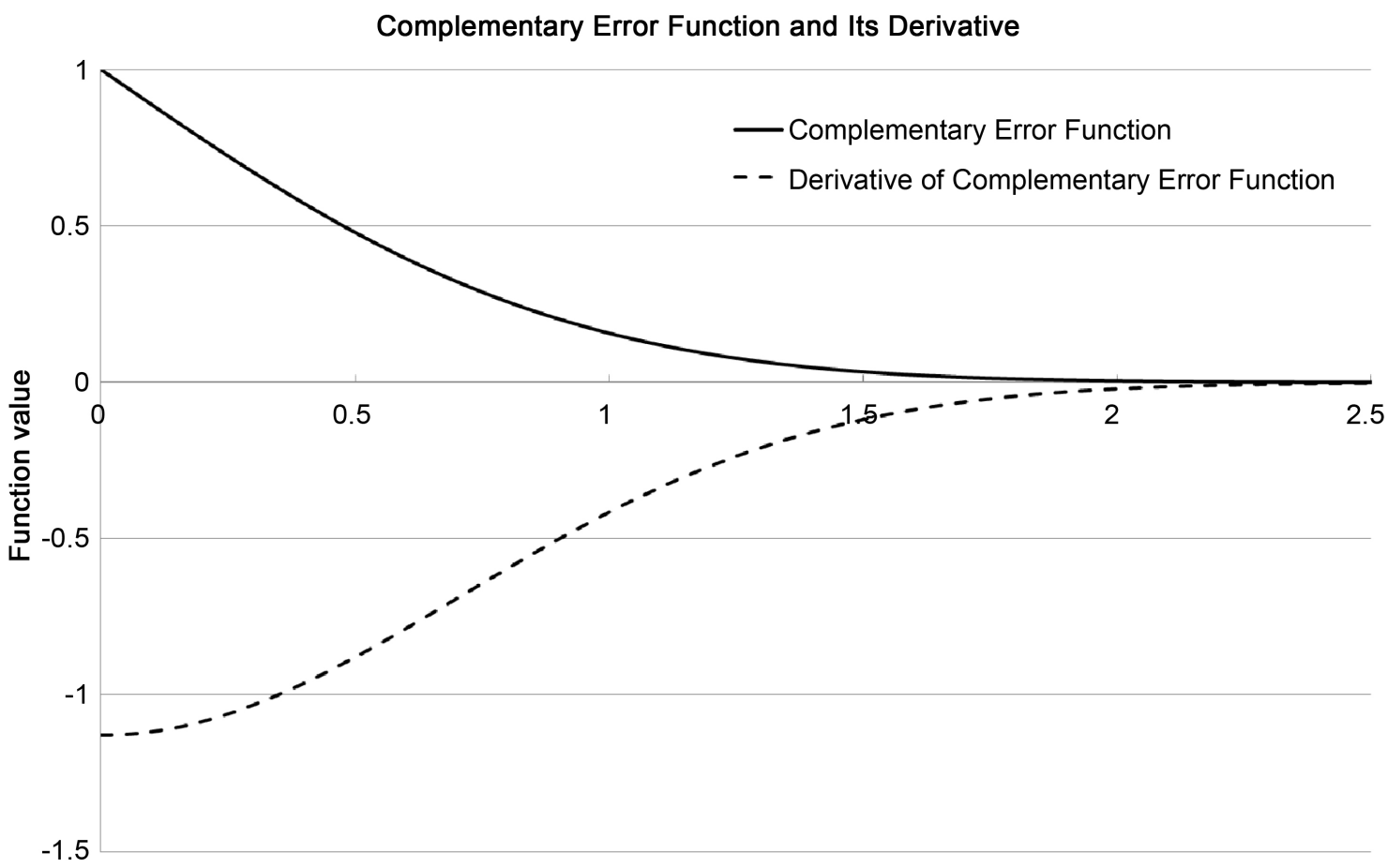

Radial Distance (u)

Figure 3. Complementary error function and its derivative.

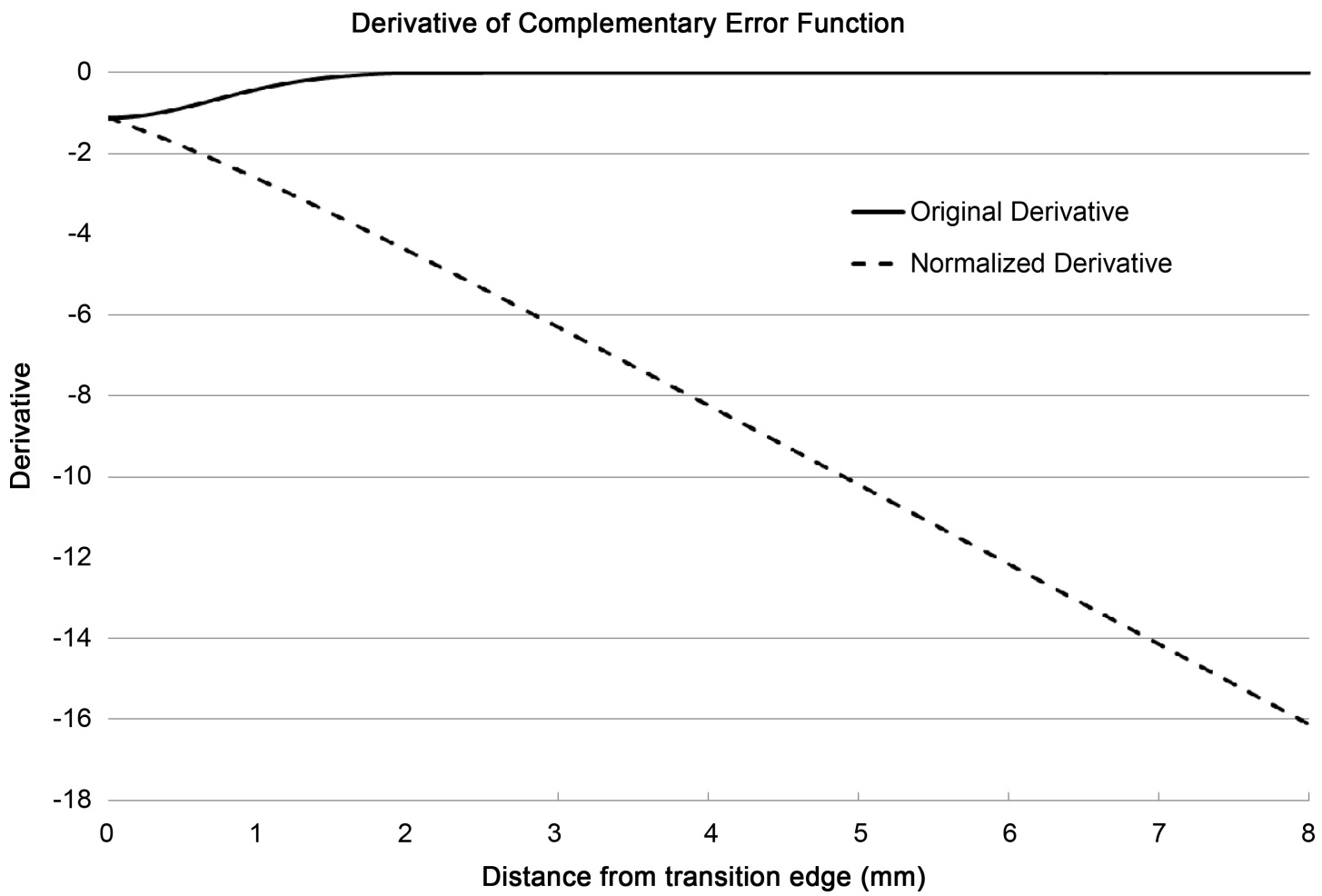

Figure 4. Normalized and unnormalized derivatives of the complementary error function. 
Sometimes, the normalized complementary error function $S(u)$ has an ending value $(u=1)$ too large when multiplied by a large transition zone height. Therefore, it is necessary to introduce an expanding factor

$$
c=\exp \left(\frac{h^{2}}{h_{0}^{2}}\right),
$$

where $h$ is the ablation depth (in microns) at the transition zone edge $(u=0)$ and $h_{0}$ (also in microns) is a constant determined by experiments. Without the use of the expanding factor, the transition curve may have unsmoothed transition. It was found that $h_{0}=55$ works well for all cases. The final transition zone function is then given by

$$
S(c \cdot u)=\frac{\operatorname{erfc}\left(u_{0}+c \cdot u\right)}{\operatorname{erfc}\left(u_{0}\right)} .
$$

It is easy to show that

$$
\frac{\partial S(c \cdot u)}{\partial u}=c \frac{\partial S(u)}{\partial u} .
$$

Hence, when the expanding factor is needed, finding $u_{0}$ should be done by equating

$$
D(u)=\frac{s}{c} .
$$

With the above-mentioned properties, the transition zone algorithm can be given as

1) For each meridian, calculate the slope at the end of the optical zone;

2) If the slope is greater than or equal to -2 , use the cubic spline function;

3) If the slope is less than -2 , use the new algorithm by

a) Calculate the expanding constant $c=\exp \left(h^{2} / 3025\right)$ where $h$ is the ablation depth at the end of the optical zone;

b) Match the slope to the normalized derivative function $D(u)=s / c$ to find $u_{0}$. An easy approximation is $u_{0}=-s /(2 c)$ (based on Figure 4);

c) Calculate the transition zone function $S(c u)=\operatorname{erfc}\left(u_{0}+c u\right) / \operatorname{erfc}\left(u_{0}\right)$ for $u=0$ to 1 ;

d) The value of $S(c u)$ replace the original spline function in areas the slope is less than -2 .

\section{Simulation Results}

To test the new algorithm, we have chosen six most typical refractive cases: spherical myopia of $-4 \mathrm{D}$, myopic astigmatism of $-4 \mathrm{DS} /-2 \mathrm{DC}$, spherical hyperopia of $+3 \mathrm{D}$, hyperopic astigmatism of $+3 \mathrm{DS} /+1 \mathrm{DC}$, mixed astigmatism of +2 DS/-3 DC, and a wavefront-based high myopic case with high order aberrations. Both the $\mathrm{x}$ - and $\mathrm{y}$-cross sections are examined. All cases use a $6 \mathrm{~mm}$ optical zone (OZ) and a $9 \mathrm{~mm}$ ablation zone (AZ) for easy comparison. The results are shown in Figure 5. 

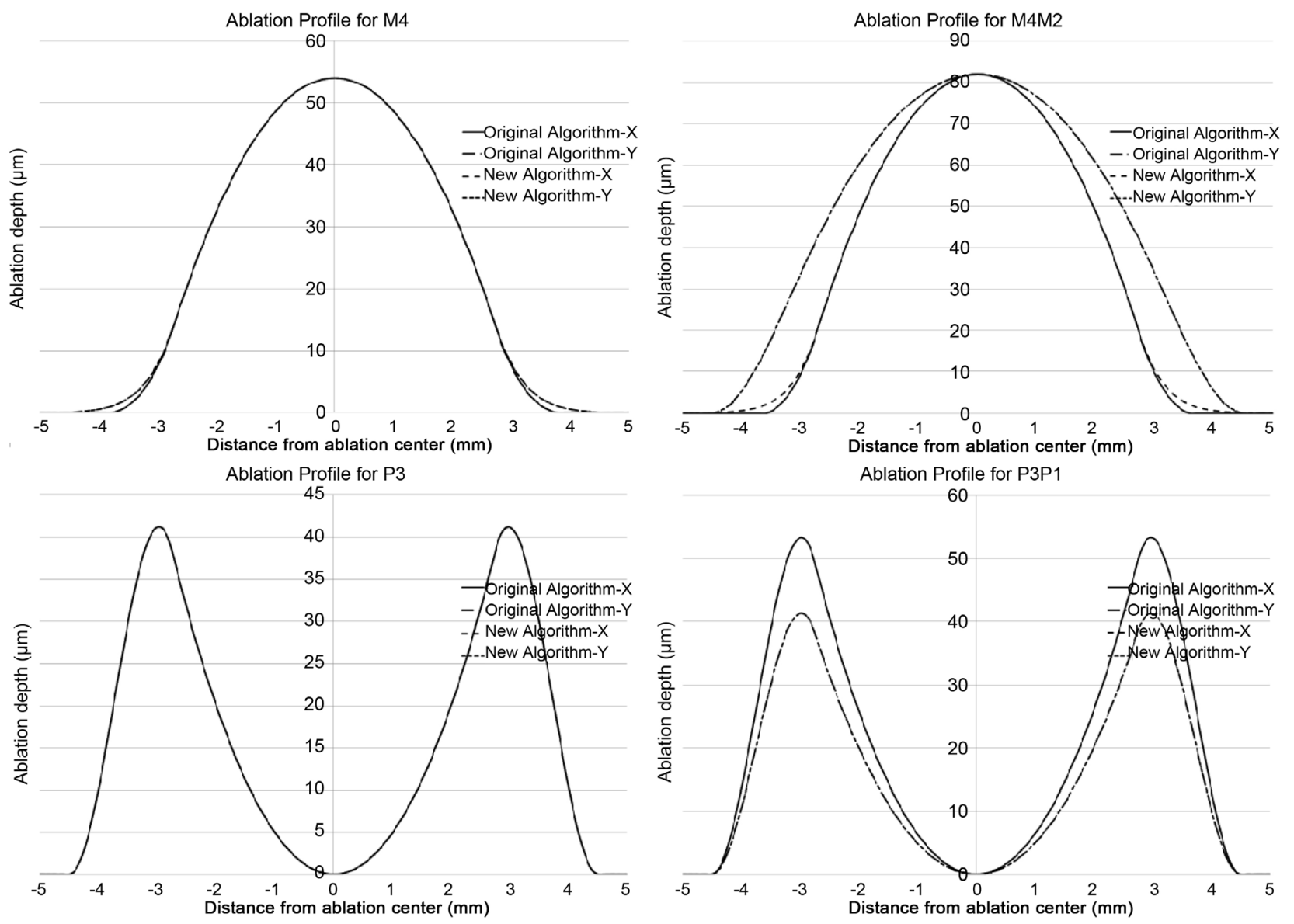

Ablation Profile for P2M3
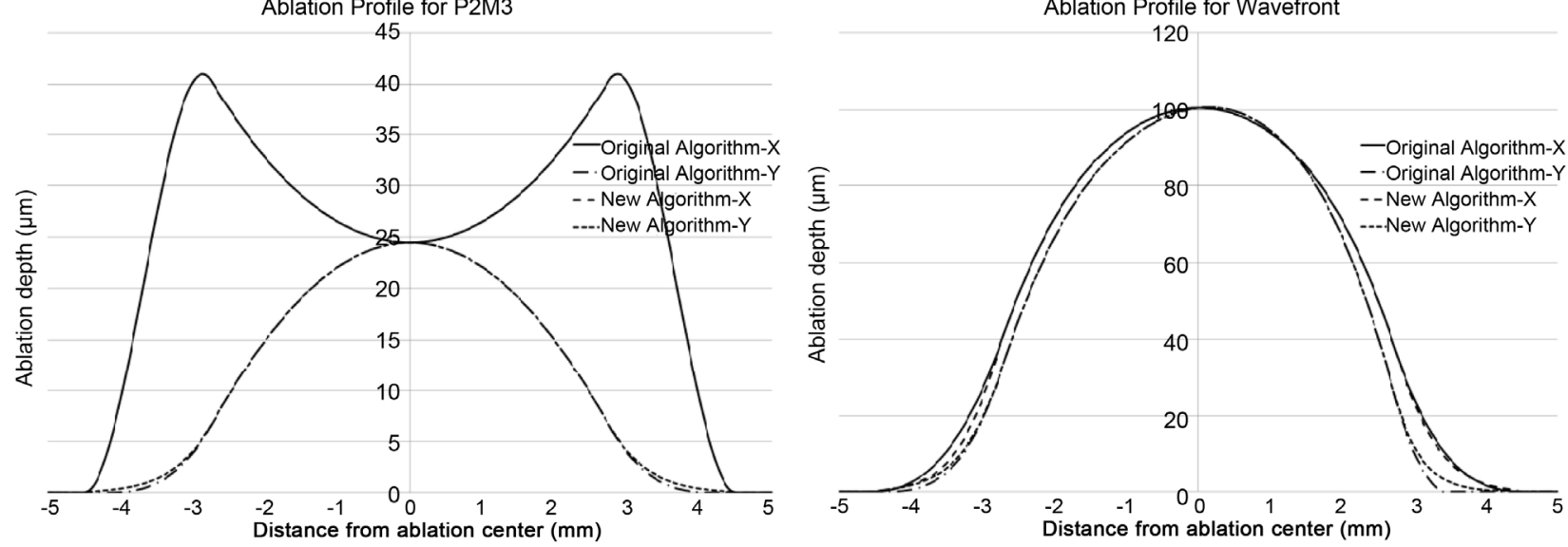

Figure 5. Cross sections of six refractive cases: myopia, myopic astigmatism, hyperopia, hyperopic astigmatism, mixed astigmatism, and wavefront based, with $6 \mathrm{~mm}$ optical zone and $9 \mathrm{~mm}$ ablation zone.

It is clear that for both hyperopia and hyperopic astigmatism, the original (cubic spline function) and the new (complementary error function) algorithms give the same result because the new algorithm uses the original cubic spline function. It is also true for the hyperopic meridian in the mixed astigmatism case. For the other cases, the new algorithm has the transition zone reached out to the $9 \mathrm{~mm}$ area while the original algorithm has the transition zone cut off early. 


\section{Conclusion}

Using cubic spline function for the purpose of transition zone for refractive surgery raises issues of running into negative values for myopic meridians when the edge of the optical zone is too steep. With the complementary error function, a normalized version appears to work very well for cases where cubic spline function does not work. Simulation shows the new algorithm is appropriate for myopic meridians where the edge of the optical zone is too steep. The result presented is useful for practical implementation of treatment algorithms in refractive surgery.

\section{References}

[1] Dai, G. (2008) Wavefront Optics for Vision Correction. SPIE Press, Bellingham, WA. https://doi.org/10.1117/3.769212

[2] Reinsch, C.H. (1967) Smoothing by Spline Functions. Numerische Mathematik, 10, 177-183. https://doi.org/10.1007/BF02162161

[3] Cook, E.R. and Peters, K. (1981) The Smoothing Spline: A New Approach to Standardizing Forest Interior Tree-Ring Width Series for Dendroclimatic Studies. Tree-Ring Bulletin, 41, 45-53.

[4] Wold, S. (1974) Spline Functions in Data Analysis. Technometrics, 16, 1-11. https://doi.org/10.1080/00401706.1974.10489142

[5] Fowler, A.H. (1966) Cubic Spline, a Curve Fitting Routine. Corp. Rep. Y-1400 (Rev. 1), Union Carbide.

[6] Dierckx, P. (1995) Curve and Surface Fitting with Splines. Oxford Science Publications, Oxford.

[7] Burt, P.J. and Adelson, E.H. (1983) A Multiresolution Spline with Application to Image Mosaics.

[8] Klein, S.A. and Barsky, B.A. (1995) Method for Generating the Anterior Surface of an Aberration-Free Contact Lens for an Arbitrary Posterior Surface. Optometry \& Vision Science, 72, 816-820. https://doi.org/10.1097/00006324-199511000-00007

[9] Barsky, B.A. (2001) Computer Aided Contact Lens Design and Fabrication Using Spline Surfaces. US Patent 6,241,355, 5 June 2001.

[10] Hohmeyer, M.E. and Barsky, B.A. (1989) Rational Continuity: Parametric, Geometric, and Frenet Frame Continuity of Rational Curves. ACM Transactions on Graphics, 8, 335-359. https://doi.org/10.1145/77269.77274

[11] Abramowitz, M. and Stegun, I.A. (1972) Handbook of Mathematical Functions with Formulas, Graphs, and Mathematical Tables, 9th Printing. Dover, New York. 
Submit or recommend next manuscript to SCIRP and we will provide best service for you:

Accepting pre-submission inquiries through Email, Facebook, LinkedIn, Twitter, etc. A wide selection of journals (inclusive of 9 subjects, more than 200 journals)

Providing 24-hour high-quality service

User-friendly online submission system

Fair and swift peer-review system

Efficient typesetting and proofreading procedure

Display of the result of downloads and visits, as well as the number of cited articles Maximum dissemination of your research work

Submit your manuscript at: http://papersubmission.scirp.org/

Or contact jamp@scirp.org 